\title{
Prognostic value of the C-reactive protein to albumin ratio: a novel inflammation-based prognostic indicator in osteosarcoma
}

This article was published in the following Dove Press journal:

OncoTargets and Therapy

2 November 2017

Number of times this article has been viewed

\author{
Yong-Jiang $\mathrm{Li}^{1, *}$ \\ Kai Yao ${ }^{2, *}$ \\ Min-Xun Lu \\ Wen-Biao Zhang' \\ Cong Xiao ${ }^{2}$ \\ Chong-Qi Tu \\ 'Department of Oncology, \\ ${ }^{2}$ Department of Orthopedics, West \\ China Hospital, Sichuan University, \\ Chengdu, People's Republic of China \\ *These authors contributed equally \\ to this work
}

\begin{abstract}
The prognostic role of the C-reactive protein to albumin ratio (CRP/Alb ratio) in patients with osteosarcoma has not been investigated. A total of 216 osteosarcoma patients were enrolled in the study. Univariate and multivariate survival analyses between the groups were performed and Kaplan-Meier analysis was conducted to plot the survival curves. Receiver operating characteristic curves were generated and areas under the curve (AUCs) were compared to assess the discriminatory ability of the inflammation-based indicators, including CRP/Alb ratio, Glasgow prognostic score (GPS), neutrophil-lymphocyte ratio (NLR), and plateletlymphocyte ratio (PLR). The optimal cutoff value was 0.210 for CRP/Alb ratio with a Youden index of 0.319 . Higher values of CRP/Alb ratio were significantly associated with poorer overall survival in univariate $(\mathrm{HR}=2.62,95 \% \mathrm{CI}=1.70-4.03 ; P<0.001)$ and multivariate $(\mathrm{HR}=2.21$, 95\% $\mathrm{CI}=1.40-3.49 ; P=0.001)$ analyses. In addition, the $\mathrm{CRP} / \mathrm{Alb}$ ratio had significantly higher AUC values compared with GPS $(P=0.003)$, NLR $(P<0.001)$, and PLR $(P<0.001)$. The study demonstrated that the $\mathrm{CRP} / \mathrm{Alb}$ ratio is an effective inflammation-based prognostic indicator in osteosarcoma, which potentially has a discriminatory ability superior to that of other inflammatory indicators including GPS, NLR, and PLR.
\end{abstract}

Keywords: osteosarcoma, CRP to albumin ratio, prognosis

\section{Introduction}

Osteosarcoma is a highly malignant bone tumor that predominantly affects adolescents and young adults, which accounts for $\sim 45 \%$ of all bone sarcomas. ${ }^{1}$ With combined modality treatment, the 5-year overall survival (OS) rate approaches $\sim 50 \%-70 \%$. Conventional prognostic factors including the presence of metastasis, tumor grade, tumor site, histological subtype, and Enneking stage have gradually exposed their inaccuracy and inadequacy in clinical practice. ${ }^{2}$ Identification of new effective prognostic factors would be valuable to stratify patients for different treatment protocols and improve the survival condition.

Cancer-related inflammation has been recognized as the 7 th hallmark of cancer, which is tightly linked to the genetic instability in cancer cells. ${ }^{3}$ Besides, the smoldering inflammation in tumor microenvironment could contribute to the progression of cancer via promoting proliferation, angiogenesis, and metastasis; reducing response to hormones and chemotherapy agents; and subverting adaptive immunity. ${ }^{3,4}$ Targeting of inflammatory pathways has been indicated as a novel way to further enhance the therapeutic efficacy. ${ }^{4,5}$ Because of the association between inflammation and cancer development, the prognostic significance of several inflammatory indicators that
Correspondence: Chong-Qi Tu Department of Orthopedics, West China Hospital, Sichuan University, No 37 Guoxuexiang, Chengdu 61004I, People's Republic of China

Email tuchongqi@outlook.com 
measure the state of systemic inflammation response has been suggested, including neutrophil-lymphocyte ratio (NLR), platelet-lymphocyte ratio (PLR), and the Glasgow prognostic score (GPS). Indeed, these indicators were reported to be linked with prognosis in several types of cancers, such as breast, lung, ovarian, and gastric cancers. ${ }^{6-9}$

Recently, a new inflammatory indicator derived from the GPS, the C-reactive protein to albumin ratio (CRP/Alb ratio), was introduced as a novel and promising prognostic marker in cancer patients, ${ }^{10,11}$ meanwhile exerting an even more effective prognostic significance and discriminatory ability than other inflammatory indicators. However, little is known about the prognostic role of these inflammation-based biomarkers, especially for the CRP/Alb, in osteosarcoma. Therefore, we conducted the current retrospective study to investigate and compare the prognostic significance of different inflammatory indicators in patients with osteosarcoma, including the CRP/Alb ratio, NLR, PLR, and GPS.

\section{Patients and methods}

The research protocol of this retrospective study was examined and approved by the institutional review board and the medical ethics committee of West China Hospital, Sichuan University.

We reviewed the electronic medical records of all patients with osteosarcoma enrolled in our hospital from June 2010 to November 2016. Patients were included if they 1) had pathologically confirmed osteosarcoma; 2) did not receive any anti-cancer treatment before; 3) had complete medical records and laboratory reports; and 4) had regular follow-up and the corresponding clinical data. The patients in the following conditions were excluded: 1) those alive with a follow-up of $<10$ months; 2) who already had neutrophilia, high procalcitonin, or high CRP or those with clinical evidence of infection or other inflammatory diseases before the first treatment; and 3) those with records of nonsteroidal anti-inflammatory drugs treatment because it could affect the results of blood test.

Clinical data of interest were extracted independently by two authors. A Microsoft Excel sheet (Microsoft Corporation, Redmond, WA, USA) was designed to collect the following records: 1) details of patients and tumor, including sex, age, tumor location, Enneking stage, and presence of pathological fracture; 2) treatment method including neoadjuvant chemotherapy and surgical option (limb salvage or amputation); 3) outcome data including the occurrence of local recurrence and metastasis and survival time; and 4) laboratory test results including Alb, CRP, neutrophil count, platelet count, and lymphocyte count that are used to calculate $\mathrm{CRP} / \mathrm{Alb}$ ratio, $\mathrm{NLR}$, and $\mathrm{PLR}$. The $\mathrm{CRP} / \mathrm{Alb}$ ratio was calculated by dividing the CRP level by the Alb level, the NLR was calculated by dividing the neutrophil count by the lymphocyte count, and the PLR was calculated by dividing the platelet count by the lymphocyte count. The GPS was allocated based on the combination of both CRP and Alb levels: patients with elevated levels of CRP $(>10 \mathrm{mg} / \mathrm{L})$ and reduced levels of $\mathrm{Alb}(\leq 35 \mathrm{~g} / \mathrm{L})$ were allocated a score of 2 , those with only one of the biochemical abnormalities were allocated a score of 1 , and those with neither of the abnormalities were allocated a score of 0 .

Follow-up protocols were formulated and performed according to the National Comprehensive Cancer Network practice guidelines. The OS, which is the main end point, was calculated from the date of initial histological diagnosis to the date of the latest follow-up of this study (November 2016) or death. The intervals of follow-up were every 3 months for the first 4 years and then every 6 months until the latest follow-up. The routine examinations of the follow-up included physical examination, radiographs of the surgical site, chest computed tomography, and laboratory tests. Bone scans were performed every 6 months.

The statistical analyses were conducted by using SPSS Statistics Version 22.0 (IBM Corporation, Chicago, IL, USA). Differences in groups were compared by using the $\chi^{2}$ test. Receiver operating characteristic (ROC) analyses were performed with OS as the end point, and the optimal cutoff values for continuous prognostic variables (ie, CRP/ Alb, NLR, and PLR) were determined at the point of the maximal Youden index. ${ }^{10,12}$ The survival curves were generated by using Kaplan-Meier analysis and evaluated by log rank test. The univariate and multivariate analyses were performed by Cox regression model to determine the independent prognostic factors. Areas under the curve (AUCs) of the ROCs were calculated and compared by Z-test to evaluate the discriminatory ability of the inflammation-based prognostic variables. ${ }^{11,13}$ All the statistical analyses were considered significant at two-tailed $P<0.05$.

\section{Ethical approval}

All the procedures performed in studies involving human participants were in accordance with the ethical standards of the medical ethics committee of West China Hospital, Sichuan University, and with the 1964 Declaration of Helsinki and its later amendments.

\section{Informed consent}

Written informed consent was obtained from all individual participants included in the study. 


\section{Results}

\section{Characteristics of patients and optimal cutoff values of inflammation-based variables}

Table 1 presents the clinicopathological characteristics of the patients. A total of 216 patients were enrolled in the current study, including 122 men and 94 women. The median age was 17 years, and majority of the tumors were located in extremities (90.3\%). In total, 184 patients were in Enneking stage I/II ( $85.2 \%)$, and the rest 32 patients were in stage III $(14.8 \%)$. Pathological fracture was presented in 18 patients $(8.3 \%)$. The neoadjuvant chemotherapy was given to 179 patients $(82.9 \%)$, and all the enrolled patients received adjuvant chemotherapy after surgery.

The optimal cutoff value for CRP/Alb ratio was calculated to be 0.210 with the Youden index of 0.319 , that for NLR was 2.65 (Youden index $=0.228$ ), and that for PLR

Table I Clinicopathological characteristics of patients

\begin{tabular}{|c|c|c|c|c|}
\hline Variables & Patients (n) & $\begin{array}{l}\text { CRP/Alb } \\
\text { ratio } \\
<0.210\end{array}$ & $\begin{array}{l}\text { CRPIAlb } \\
\text { ratio } \\
\geq 0.210\end{array}$ & P-value \\
\hline Total patients & 216 & 95 & 121 & - \\
\hline \multicolumn{4}{|l|}{ Gender } & 0.230 \\
\hline Male & 122 & 58 & 64 & \\
\hline Female & 94 & 37 & 57 & \\
\hline \multicolumn{4}{|l|}{ Age (years) } & 0.259 \\
\hline$<17$ & 98 & 39 & 59 & \\
\hline$\geq 17$ & 118 & 56 & 62 & \\
\hline \multicolumn{4}{|l|}{ Tumor location } & 0.082 \\
\hline Extremities & 195 & 82 & 113 & \\
\hline Nonextremities & 21 & 13 & 8 & \\
\hline \multicolumn{4}{|l|}{ Enneking stage } & 0.006 \\
\hline I/II & 184 & 88 & 96 & \\
\hline III & 32 & 7 & 25 & \\
\hline \multicolumn{4}{|c|}{ Pathological fracture } & 0.148 \\
\hline Yes & 18 & 5 & 13 & \\
\hline No & 198 & 90 & 108 & \\
\hline \multicolumn{4}{|c|}{ Treatment with NACT } & 0.236 \\
\hline Yes & 179 & 82 & 97 & \\
\hline No & 37 & 13 & 24 & \\
\hline Body mass index & - & 20.2 & 19.3 & 0.031 \\
\hline \multicolumn{5}{|l|}{$\left(\mathrm{kg} / \mathrm{m}^{2}\right.$, mean $)$} \\
\hline NLR & & & & 0.003 \\
\hline$<2.65$ & 123 & 65 & 58 & \\
\hline$\geq 2.65$ & 93 & 30 & 63 & \\
\hline \multicolumn{4}{|l|}{ PLR } & 0.005 \\
\hline$<118$ & 75 & 43 & 32 & \\
\hline$\geq 118$ & $14 \mid$ & 52 & 89 & \\
\hline \multicolumn{4}{|l|}{ GPS } & $<0.00$ I \\
\hline 0 & 128 & 72 & 56 & \\
\hline 1 & 77 & 23 & 54 & \\
\hline 2 & II & 0 & II & \\
\hline
\end{tabular}

Abbreviations: CRP/Alb ratio, C-reactive protein to albumin ratio; GPS, Glasgow prognostic score; NACT, neoadjuvant chemotherapy; NLR, neutrophil-lymphocyte ratio; PLR, platelet-lymphocyte ratio. was 118 (Youden index $=0.204$ ). Table 1 presents the clinicopathological significance of the CRP/Alb ratio. The $\mathrm{CRP} / \mathrm{Alb}$ ratio was found to be correlated with tumor stage ( $P=0.006)$, body mass index (BMI; $P=0.031$ ), and the other inflammatory indicators (NLR, $P=0.003$; PLR, $P=0.005$; and GPS, $P<0.001)$.

\section{Survival analysis}

The median OS of all cohorts was 31.5 months, and 112 (51.9\%) patients were alive at the end of the follow-up. The survival curves revealed that patients with lower CRP/Alb ratio had significantly better survival than those with higher $\mathrm{CRP} /$ Alb ratio $(P<0.001$; Figure $1 \mathrm{~A})$. Similar results were also observed when patients were grouped by $\operatorname{NLR}(P=0.003$; Figure 1B), PLR ( $P=0.012$; Figure $1 \mathrm{C})$, and GPS ( $P=0.001$; Figure 1D).

Table 2 presents the univariate and multivariate analysis results. The univariate analysis revealed that variables associated with better OS included tumor site (HR $=1.86,95 \%$ $\mathrm{CI}=1.06-3.27 ; P=0.031)$, metastasis $(\mathrm{HR}=2.74,95 \%$ $\mathrm{CI}=1.75-4.29 ; P<0.001)$, and the inflammation-based indicators including PLR ( $\mathrm{HR}=1.76,95 \% \mathrm{CI}=1.13-2.75$; $P=0.012)$, NLR (HR $=1.81,95 \% \mathrm{CI}=1.23-2.67 ; P=0.003)$, GPS $(\mathrm{HR}=1.95,95 \% \mathrm{CI}=1.33-2.87 ; P=0.001)$, and $\mathrm{CRP} / \mathrm{Alb}$ ratio $(\mathrm{HR}=2.62,95 \% \mathrm{CI}=1.70-4.03 ; P<0.001)$. Because the inflammatory indicators of $\mathrm{CRP} / \mathrm{Alb}$ ratio, NLR, PLR, and GPS have correlations, only the CRP/Alb ratio was incorporated in the multivariate analysis. The multivariate analysis demonstrated that metastasis (HR $=1.99,95 \%$ $\mathrm{CI}=1.23-3.22 ; P=0.005)$ and $\mathrm{CRP} / \mathrm{Alb}$ ratio $(\mathrm{HR}=2.21$, $95 \% \mathrm{CI}=1.40-3.49 ; P=0.001)$ were independent prognostic factors for better OS.

\section{ROC curves of the inflammation-based variables}

ROC curves were generated for the survival status to evaluate the discriminatory ability of CRP/Alb, NLR, PLR, and GPS (Figure 2). As shown in Table 3, the AUCs were 0.680 $(95 \% \mathrm{CI}=0.614-0.742)$ for $\mathrm{CRP} / \mathrm{Alb}$ ratio, $0.644(95 \%$ $\mathrm{CI}=0.577-0.708)$ for NLR, $0.620(95 \% \mathrm{CI}=0.552-0.685)$ for GPS, and 0.609 ( $95 \% \mathrm{CI}=0.540-0.674)$ for PLR. The $\mathrm{CRP} / \mathrm{Alb}$ ratio had significantly higher AUC values compared with NLR $(P<0.001)$, GPS $(P=0.003)$, and PLR $(P<0.001$; Table 3$)$.

\section{Discussion}

To our knowledge, this retrospective analysis based on individual data from 216 osteosarcoma patients first explored the prognostic value of $\mathrm{CRP} / \mathrm{Alb}$ ratio and compared it with 
A

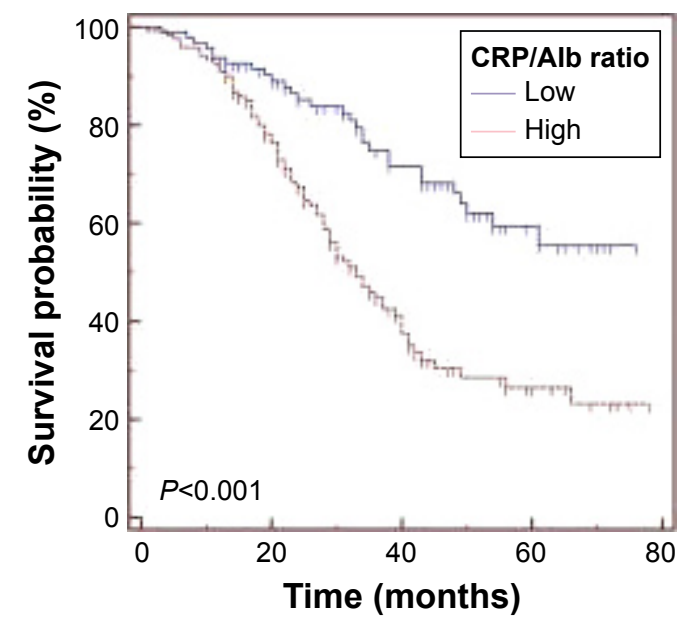

C

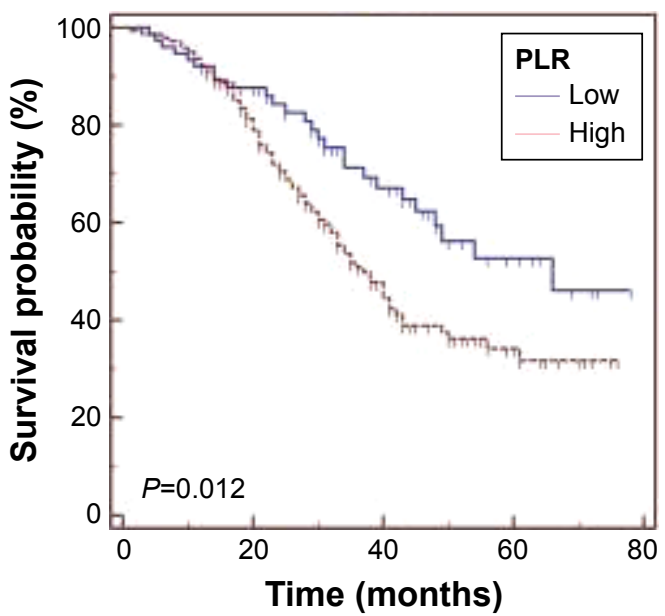

B

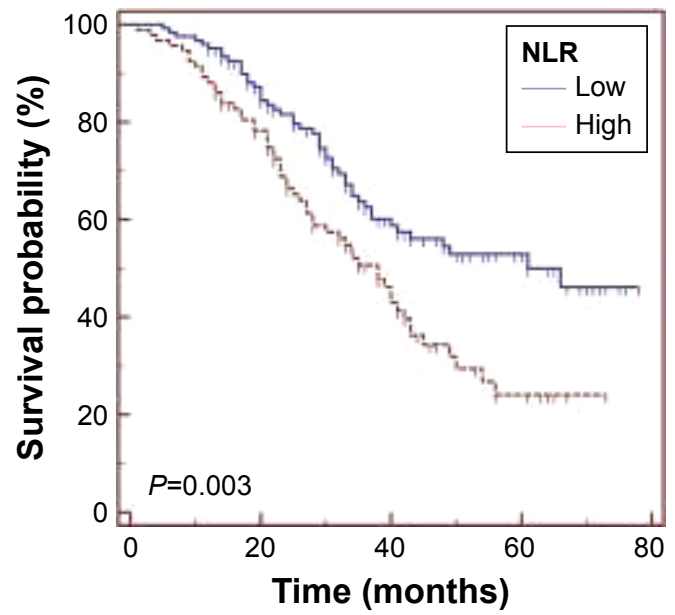

D

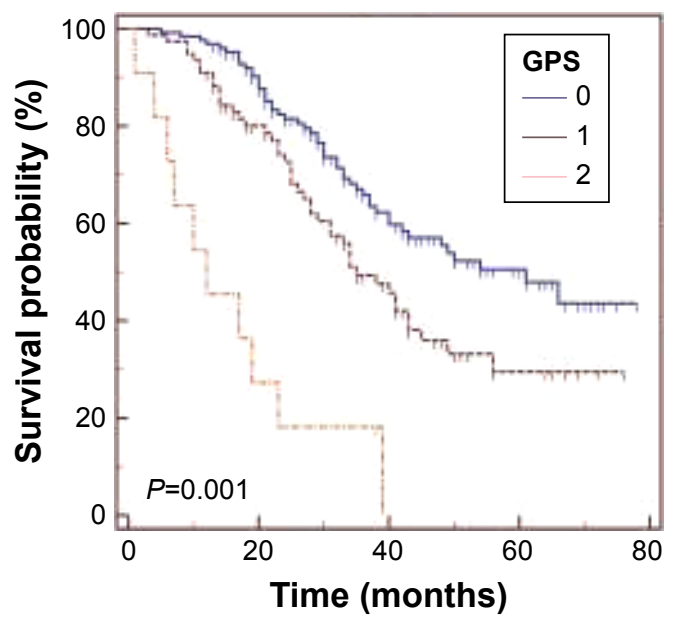

Figure I Kaplan-Meier survival curves for overall survival in 216 osteosarcoma patients according to (A) CRP/Alb ratio, (B) NLR, (C) PLR, and (D) the GPS. Abbreviations: CRP/Alb ratio, C-reactive protein to albumin ratio; GPS, Glasgow prognostic score; NLR, neutrophil to lymphocyte ratio; PLR, platelet to lymphocyte ratio.

other inflammatory indicators in patients with osteosarcoma. Our findings demonstrated that the CRP/Alb ratio could serve as a novel and promising inflammatory prognostic indicator in osteosarcoma and has a discriminatory ability even superior to that of other inflammatory indicators including GPS, NLR, and PLR.

It is becoming increasingly recognized that the systematic inflammatory response plays a crucial role in the development

Table 2 Univariate and multivariate analyses of overall survival using the Cox proportional hazard model

\begin{tabular}{|c|c|c|c|c|c|c|}
\hline \multirow[t]{2}{*}{ Variables } & \multicolumn{3}{|c|}{ Univariate analysis } & \multicolumn{3}{|c|}{ Multivariate analysis } \\
\hline & HR & $95 \% \mathrm{Cl}$ & $P$-value & HR & $95 \% \mathrm{Cl}$ & $P$-value \\
\hline Gender (male vs female) & 1.18 & $0.79-1.75$ & 0.401 & & & \\
\hline Age (<17 vs $\geq 17$ years $)$ & 1.27 & $0.86-1.87$ & 0.227 & & & \\
\hline Pathological fracture (yes vs no) & 1.29 & $0.67-2.48$ & 0.463 & & & \\
\hline Tumor site (extremities vs nonextremities) & 1.86 & $1.06-3.27$ & 0.031 & 1.63 & $0.92-2.88$ & 0.094 \\
\hline Metastasis & 2.74 & $1.75-4.29$ & $<0.001$ & 1.99 & $1.23-3.22$ & 0.005 \\
\hline Neoadjuvant chemotherapy (no vs yes) & 1.40 & $0.87-2.24$ & 0.162 & & & \\
\hline PLR $(\geq 118$ vs $<118)$ & 1.76 & $1.13-2.75$ & 0.012 & & & \\
\hline NLR $(\geq 2.65$ vs $<2.65)$ & 1.81 & $1.23-2.67$ & 0.003 & & & \\
\hline GPS (I/2 vs 0$)$ & 1.95 & $1.33-2.87$ & 0.001 & & & \\
\hline CRP/Alb ratio $(\geq 0.210$ vs $<0.210)$ & 2.62 & $1.70-4.03$ & $<0.001$ & 2.21 & $1.40-3.49$ & 0.001 \\
\hline
\end{tabular}

Abbreviations: CRP/Alb ratio, C-reactive protein to albumin ratio; GPS, Glasgow prognostic score; NLR, neutrophil-lymphocyte ratio; PLR, platelet-lymphocyte ratio. 


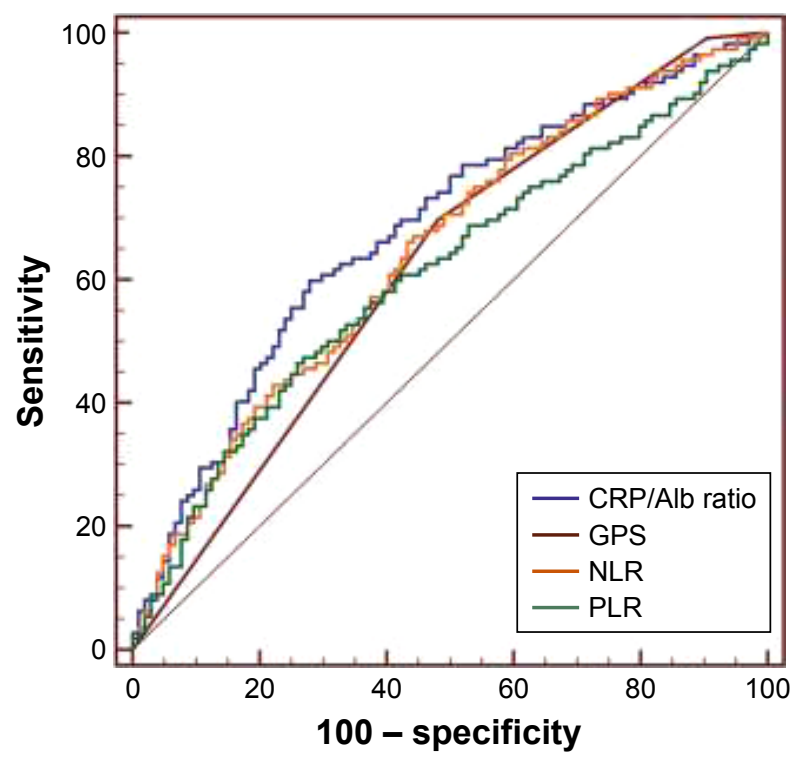

Figure 2 Comparison of the areas under the ROC curves for prognosis among the inflammation-based prognostic indicators.

Abbreviations: CRP/Alb ratio, C-reactive protein to albumin ratio; GPS, Glasgow prognostic score; NLR, neutrophil to lymphocyte ratio; PLR, platelet to lymphocyte ratio; ROC, receiver operating characteristic.

and progression of cancers. ${ }^{3,4}$ Mechanisms for the inflammatory response in cancer have been proposed: the growth and invasion of tumor accompany with tumor necrosis, hypoxia, and local tissue damage, disrupting the homeostasis and inciting acute-phase responses. ${ }^{14,15}$ Then, tumor cells and/or tumor-associated leukocytes could release pro-inflammatory cytokines, including IL-1, IL-6, IL-8, tumor necrosis factor, and vascular endothelial growth factor. ${ }^{16-18}$ These inflammatory cytokines could promote tumor angiogenesis, growth, invasion, and metastasis; subvert adaptive immune response; and enhance the resistance to cytotoxic agents. ${ }^{19,20}$

$\mathrm{CRP}$ is an acute-phase reactant that is regulated by proinflammatory cytokines, especially IL-6. The presence of systematic inflammatory status in cancer, reflected by elevated CRP level, often accompanies with decreased serum Alb concentration, lean tissue consuming, and continuous weight

Table 3 Comparison of the AUCs between inflammation-based prognostic indicators

\begin{tabular}{lllll}
\hline Variables & AUC & $\mathbf{9 5 \%} \mathbf{C l}$ & $\boldsymbol{P}$-value & $\boldsymbol{P}$-value* \\
\hline CRP/Alb ratio & 0.680 & $0.614-0.742$ & $<0.001$ & - \\
NLR & 0.644 & $0.577-0.708$ & $<0.001$ & $<0.00 \mathrm{I}$ \\
GPS & 0.620 & $0.552-0.685$ & $0.00 \mathrm{I}$ & 0.003 \\
PLR & 0.609 & $0.540-0.674$ & 0.004 & $<0.00 \mathrm{I}$ \\
\hline
\end{tabular}

Note: *Comparison of AUC values between the CRP/Alb ratio and other inflammation-based prognostic indicators using Z-test method.

Abbreviations: AUC, area under the curve; CRP/Alb ratio, C-reactive protein to albumin ratio; GPS, Glasgow prognostic score; NLR, neutrophil-lymphocyte ratio; PLR, platelet-lymphocyte ratio. loss, resulting in an impaired nutritional status and increasing the mortality. ${ }^{18,21}$ Indeed, elevated CRP level was found to be correlated with unfavorable prognosis in osteosarcoma patients. ${ }^{22-24}$ However, neutrophils interact with tumor cells via secreting cytokines, promoting tumor development. ${ }^{25,26}$ Lymphocytes play a crucial role in mediating the immunologic destruction of cancer cells, and lymphocytopenia indicates impairment of the host immune response to tumor. ${ }^{27,28}$ Platelets were also reported to serve as chemoattractants, contributing to the migration of tumor cells. ${ }^{29,30}$ These inflammatory theoretic backgrounds lead to the emerging of several inflammation-based prognostic indicators in cancer patients, including NLR, PLR, and GPS.

The CRP/Alb ratio is an indicator derived from GPS. Instead of only stratified into three scores $(0,1$, and 2$)$ in GPS, the CRP/Alb ratio is calculated as a quantitative ratio with a continuous range of values, which may theoretically better utilize the values of CRP and Alb. However, studies focusing on CRP/Alb ratio in cancer patients are limited, and no relevant studies have been reported in patients with osteosarcoma. In the present study, we aimed to evaluate the clinicopathological and prognostic significance of the $\mathrm{CRP} / \mathrm{Alb}$ ratio in osteosarcoma and compared it with other inflammatory indicators.

We found that the CRP/Alb ratio is statistically associated with Enneking stage, BMI, and other inflammatory indicators (NLR, PLR, and GPS). In the univariate analysis, high values of all the four inflammation indicators were found to be significantly associated with the poor prognosis of osteosarcoma patients. Besides, the $\mathrm{CRP} / \mathrm{Alb}$ ratio remained an independent prognostic factor in the multivariate analysis. The results were in accordance with the studies investigating $\mathrm{CRP} / \mathrm{Alb}$ ratio in other types of tumors. ${ }^{10,11}$

In the ROC analysis, we compared the prognostic value of $\mathrm{CRP} / \mathrm{Alb}$ ratio with other inflammatory indicators by utilizing the $Z$-test, which has been used and validated in previous studies. ${ }^{11,13}$ The AUC value of CRP/Alb ratio was significantly higher than that of NLR, PLR, and GPS, indicating a superior discriminatory ability of $\mathrm{CRP} / \mathrm{Alb}$ ratio than other inflammatory indicators in terms of prognosis in osteosarcoma patients. The results demonstrated that, compared with the GPS that separately utilizes the values of CRP and $\mathrm{Alb}$, the CRP/Alb ratio in the form of continuous variable could more rationally integrate these values and could acquire more accurate prognostic information.

We also identified the tumor site as a prognostic indicator in the univariate analysis, and this is possibly due to the fact that the site of origin could critically influence chances 
for radical surgery, which could greatly influence the treatment outcomes and survival. In the multivariate analysis, the metastasis also remained an independent prognostic factor. Compared with these prognostic indicators, the CRP/Alb ratio is a simple, easily available, economical, objective, and reproducible indicator and could be measured at the early start of treatment and monitored throughout the entire therapy period, which make it potentially easy and worthy to be promoted in clinical practice.

It needs to be noted that lower BMI levels were found to be correlated with higher values of $\mathrm{CRP} / \mathrm{Alb}$ ratio, which supports the view that cancer-related systematic inflammation often accompanies with an elevation in the overall consumption status of the body. Thus, the $\mathrm{CRP} / \mathrm{Alb}$ ratio may not only be an inflammatory indicator, but also be a marker reflecting the nutritional status of cancer patients. There have been studies using nutritional supplements to alter the inflammatory and nutritional status and improve the immune function of cancer patients. ${ }^{31,32}$ The application of utilizing CRP/Alb ratio in evaluating the inflammatory and trophic status and assisting nutritional support therapy of cancer patients may be a promising way for future relevant researches.

The CRP/Alb ratio is based on two protein components, and the NLR and PLR are based on two cellular components. Although we found that the prognostic ability of CRP/Alb ratio may be superior to that of the NLR or PLR, which suggested that the inflammation-related protein parameters might be more reliable than the cellular parameters in predicting prognosis, the two categories of indicators are actually not incompatible with each other. The complementary combination of CRP/Alb ratio with NLR and PLR may provide firmer prognostic information for clinicians. In addition, whether these inflammatory indicators could be incorporated in cancer patients' stratification system to instruct individual treatment is needed to be clarified in future prospective studies.

A potential limitation of this study is that it was a retrospective, single-center study. Although the patient samples were relatively large among the studies concerning osteosarcoma patients and the multivariate analysis showed that the $\mathrm{CRP} / \mathrm{Alb}$ ratio remained an independent prognostic factor, the value is still restricted due to its retrospective design. Second, heterogeneity existed in treatments of these patients, which may bring potential bias to the results. Thus, the results of the current study should be interpreted with caution and remained to be validated in future multicenter prospective studies.

\section{Conclusion}

Our findings demonstrated that the CRP/Alb ratio is a novel and promising inflammation-based prognostic indicator in osteosarcoma patients, which may potentially have a discriminatory ability superior to that of other inflammatory indicators including GPS, NLR, and PLR. Future multicenter prospective studies are needed to validate our findings and further explore the value of combined use of these inflammatory indicators in further prolonging survival of osteosarcoma patients.

\section{Acknowledgments}

The authors gratefully acknowledge the staff in the Department of Oncology, Department of Orthopedics, and EvidenceBased Medicine Center, West China Hospital, Sichuan University.

\section{Author contributions}

All authors contributed toward data analysis, drafting and critically revising the paper, gave final approval of the version to be published, and agree to be accountable for all aspects of the work.

\section{Disclosure}

The authors report no conflicts of interest in this work.

\section{References}

1. Mirabello L, Troisi RJ, Savage SA. Osteosarcoma incidence and survival rates from 1973 to 2004: data from the Surveillance, Epidemiology, and End Results Program. Cancer. 2009;115(7):1531-1543.

2. Li YJ, Dai YL, Cheng YS, Zhang WB, Tu CQ. Positron emission tomography (18)F-fluorodeoxyglucose uptake and prognosis in patients with bone and soft tissue sarcoma: a meta-analysis. Eur J Surg Oncol. 2016; 42(8):1103-1114.

3. Mantovani A, Allavena P, Sica A, Balkwill F. Cancer-related inflammation. Nature. 2008;454(7203):436-444.

4. Diakos CI, Charles KA, McMillan DC, Clarke SJ. Cancer-related inflammation and treatment effectiveness. Lancet Oncol. 2014;15(11): e493-e503.

5. Aggarwal BB, Vijayalekshmi RV, Sung B. Targeting inflammatory pathways for prevention and therapy of cancer: short-term friend, longterm foe. Clin Cancer Res. 2009;15(2):425-430.

6. Koh CH, Bhoo-Pathy N, Ng KL, et al. Utility of pre-treatment neutrophillymphocyte ratio and platelet-lymphocyte ratio as prognostic factors in breast cancer. Br J Cancer. 2015;113(1):150-158.

7. Gu X, Sun S, Gao XS, et al. Prognostic value of platelet to lymphocyte ratio in non-small cell lung cancer: evidence from 3,430 patients. Sci Rep. 2016;6:23893.

8. Zhu J, Wang H, Liu CC, Lu Y, Tang H. The Glasgow Prognostic Score (GPS) is a novel prognostic indicator in advanced epithelial ovarian cancer: a multicenter retrospective study. J Cancer Res Clin Oncol. 2016; 142(11):2339-2345.

9. Li Y, Wang C, Xu M, et al. Preoperative NLR for predicting survival rate after radical resection combined with adjuvant immunotherapy with CIK and postoperative chemotherapy in gastric cancer. $J$ Cancer Res Clin Oncol. 2017;143(5):861-871. 
10. Ishizuka M, Nagata H, Takagi K, Iwasaki Y, Shibuya N, Kubota K. Clinical significance of the $\mathrm{C}$-reactive protein to albumin ratio for survival after surgery for colorectal cancer. Ann Surg Oncol. 2016;23(3): 900-907.

11. Liu Z, Jin K, Guo M, et al. Prognostic value of the CRP/Alb ratio, a novel inflammation-based score in pancreatic cancer. Ann Surg Oncol. 2017;24(2):561-568.

12. Youden WJ. Index for rating diagnostic tests. Cancer. 1950;3(1): 32-35.

13. Camp RL, Dolled-Filhart M, Rimm DL. X-tile: a new bio-informatics tool for biomarker assessment and outcome-based cut-point optimization. Clin Cancer Res. 2004;10(21):7252-7259.

14. Balkwill F, Mantovani A. Inflammation and cancer: back to Virchow? Lancet. 2001;357(9255):539-545.

15. Heikkila K, Ebrahim S, Lawlor DA. A systematic review of the association between circulating concentrations of $\mathrm{C}$ reactive protein and cancer. J Epidemiol Community Health. 2007;61(9):824-833.

16. Jablonska E, Kiluk M, Markiewicz W, Piotrowski L, Grabowska Z, Jabłoński J. TNF-alpha, IL-6 and their soluble receptor serum levels and secretion by neutrophils in cancer patients. Arch Immunol Ther Exp (Warsz). 2001;49(1):63-69.

17. McCourt M, Wang JH, Sookhai S, Redmond HP. Activated human neutrophils release hepatocyte growth factor/scatter factor. Eur J Surg Oncol. 2001;27(4):396-403

18. McMillan DC. The systemic inflammation-based Glasgow Prognostic Score: a decade of experience in patients with cancer. Cancer Treat Rev. 2013;39(5):534-540

19. Kantola T, Klintrup K, Vayrynen JP, et al. Stage-dependent alterations of the serum cytokine pattern in colorectal carcinoma. Br J Cancer. 2012;107(10):1729-1736.

20. Shamamian P, Schwartz JD, Pocock BJ, et al. Activation of progelatinase A (MMP-2) by neutrophil elastase, cathepsin G, and proteinase-3: a role for inflammatory cells in tumor invasion and angiogenesis. $J$ Cell Physiol. 2001;189(2):197-206.

21. Roxburgh CS, McMillan DC. Cancer and systemic inflammation: treat the tumour and treat the host. Br J Cancer. 2014;110(6):1409-1412.
22. Aggerholm-Pedersen N, Maretty-Kongstad K, Keller J, Baerentzen S, Safwat A. The prognostic value of serum biomarkers in localized bone sarcoma. Transl Oncol. 2016;9(4):322-328.

23. Li X, Tian F, Wang F, Li Y. Serum C-reactive protein and overall survival of patients with osteosarcoma. Tumour Biol. 2015;36(7):5663-5666.

24. Yi JH, Wang D, Li ZY, Hu J, Niu XF, Liu XL. C-reactive protein as a prognostic factor for human osteosarcoma: a meta-analysis and literature review. PLoS One. 2014;9(5):e94632.

25. Ji H, Houghton AM, Mariani TJ, et al. K-ras activation generates an inflammatory response in lung tumors. Oncogene. 2006;25(14) 2105-2112.

26. Swierczak A, Mouchemore KA, Hamilton JA, Anderson RL. Neutrophils: important contributors to tumor progression and metastasis. Cancer Metastasis Rev. 2015;34(4):735-751.

27. Li MX, Liu XM, Zhang XF, et al. Prognostic role of neutrophil-tolymphocyte ratio in colorectal cancer: a systematic review and metaanalysis. Int J Cancer. 2014;134(10):2403-2413.

28. Lissoni P, Brivio F, Fumagalli L, et al. Efficacy of cancer chemotherapy in relation to the pretreatment number of lymphocytes in patients with metastatic solid tumors. Int J Biol Markers. 2004;19(2):135-140.

29. Ishikawa S, Miyashita T, Inokuchi M, et al. Platelets surrounding primary tumor cells are related to chemoresistance. Oncol Rep. 2016; 36(2):787-794.

30. Orellana R, Kato S, Erices R, et al. Platelets enhance tissue factor protein and metastasis initiating cell markers, and act as chemoattractants increasing the migration of ovarian cancer cells. BMC cancer. 2015; 15:290.

31. Mocellin MC, Camargo CQ, Nunes EA, Fiates GMR, Trindade EBSM. A systematic review and meta-analysis of the $n-3$ polyunsaturated fatty acids effects on inflammatory markers in colorectal cancer. Clin Nutr. 2016;35(2):359-369.

32. Mocellin MC, Pastore e Silva Jde A, Camargo Cde Q, et al. Fish oil decreases C-reactive protein/albumin ratio improving nutritional prognosis and plasma fatty acid profile in colorectal cancer patients Lipids. 2013;48(9):879-888.
OncoTargets and Therapy

\section{Publish your work in this journal}

OncoTargets and Therapy is an international, peer-reviewed, open access journal focusing on the pathological basis of all cancers, potential targets for therapy and treatment protocols employed to improve the management of cancer patients. The journal also focuses on the impact of management programs and new therapeutic agents and protocols on

\section{Dovepress}

patient perspectives such as quality of life, adherence and satisfaction. The manuscript management system is completely online and includes a very quick and fair peer-review system, which is all easy to use. Visit http://www.dovepress.com/testimonials.php to read real quotes from published authors. 